\title{
Cloud, network and sensing in a Smart City, toward a cloud of meshed cooperative heterogeneous things
}

\author{
Valeria Loscri, Nathalie Mitton, Riccardo Petrolo
}

\begin{abstract}
With the increase of their population, cities must adapt to offer more efficient services and better life quality. ICT and Internet of Things are among the technologies that enable smarter transportation, waste collection, energy and resource management, etc. But this does not come at no cost and new services arise through the collection and processing of a large set of distributed and ubiquitous data. This chapter discusses the requirements needed to achieve a Smart City, identifying the main technical challenges in the heterogeneity of devices, data, networks, protocols, and standards. To overcome these limitations, we propose our vision of Cloud of Meshed Cooperative heterogeneous Things (CoMCoT). CoMCoT targets to enable every traditional city's entity (node, user, and provider) to be exposed and consumed as a Service. Beyond traditional Cloud of Things (CoT) services such as data abstraction and mutualization, the CoMCoT provides more holistic functionalities by aiming at full interoperability.
\end{abstract}

\section{Introduction}

Cities are growing at a fast pace and by 2050 the $70 \%$ of the world's population is expected to be urban. In this context, it is important for cities to become "smarter" and to be ready to accommodate this huge amount of citizens and to face new arduous challenges e.g., traffic congestion, air pollution, waste management, water monitoring, etc.

In the last few decades, a lot of attention has been given to the evolution of the urban development towards technology, innovation and globalization, highlighting on the need of "smarter" urban ecosystems. The term used to describe this revolution is "Smart City".

Information and Communication Technologies (ICT) are playing a key role in the innovation process of our cities. They are indeed used to sense physical phenomena, process them, and take decisions in order to bring advantages to the citizens and the community. On the other side, all this interest results in a proliferation in terms of hardware, software, standards and protocols, making the landscape fragmented. In this context, the Cloud of Things (CoT) has been introduced as a solution to share sensed data across different applications and endusers, to provide advanced services. However, the CoT does not deal with smart network aspects.

In this chapter, we will discuss the requirements needed to achieve a Smart City. We identify the main technical challenges in the heterogeneity of devices, data, networks, protocols, and standards. To overcome these limitations, we propose our vision of Cloud of Meshed 
Cooperative heterogeneous Things (CoMCoT). CoMCoT targets to enable every traditional city's entity (node, user, and provider) to be exposed and consumed as a Service. Beyond traditional CoT services such as data abstraction and mutualization, the CoMCoT provides more holistic functionalities by targeting full interoperability.

The chapter is organized as follows: Section 2 identifies the technical challenges and use cases, Section 3 describes the Cloud of Things and its limitations in the different scenarios envisioned in this chapter and our vision of what a Smart City should provide. In Section 4, we introduce our vision of CoMCoT. In Section 5, we describe the functionalities, tools and standards requested to realize that vision. Finally, in Section 6, we argue socio-economic challenges and Section 7 concludes this chapter.

\section{Towards Smarter Cities}

"Smart city" is a recent tendency and a popular term used to describe a city that implements new tools based on Information and Communication Technologies (ICT) to provide new services to its citizens [citypulse,2014,may] . Often, people refers to "smart city" by using different synonyms, e.g., connected city, augmented city, digital city, inclusive city, etc.; however questions such as "what to expect from a smart city?", "what are the requirements to achieve a smart city?", "when and why a city can be defined smart?", etc. need to be yet answered. In the following, we define requirements and use case examples of smart cities.

In a nutshell, a smart city relies on ICT to ensure a more efficient and democratic city with the final objective to bring advantages to the citizens and the community. To this end, transformations based on smart services and fluent mobility are required to allow the city to be more sustainable, safer, respectful of the nature - by providing a better waste management, a better management and consumption of natural energy resources, etc. However, this revolution cannot be only driven by technology, it should be fostered by citizens and the different authorities. To be successful and adopted, all the deployed technologies must ensure citizen's privacy and security without being intrusive. On the other side, levers to make citizen to adopt and use a given technology or tool are not universal and are greatly influenced by socio-economical and cultural contexts, as we will discuss in Section 6. Tools and digital applications intended to make a city smart(er) must meet different requirements, technological, societal, and ethical.

\section{Technological requirements}

ICT plays a key role in the smart city revolution. From a technological perspective, to achieve smart city services, there is a need to address the following requirements:

- Data should be collected on specific locations of the city to catch the city's pulse;

- Network standards for data collection and self-organization, need to be redesigned and deployed;

- Servers, clouds, and databases are needed for storing data and executing specific algorithms to process data; 
- Data visualization and interpretation tools are needed to provide real-time feedbacks to citizens and different city stakeholders.

Let's now detail these components.

\section{Data sources and content}

Due to the city complexity, collected data may be heterogeneous and sensed through different means. For example, data can be gathered via a set of sensors distributed over the city, which sense physical and environmental information such as temperature, air quality, humidity, light, etc. At the same time, data relative to human mobility can be collected by using a counter of passages or visitors (such as access control for public transportation or buildings), mobile applications, and CCTV cameras.

The above examples emphasize the high level of heterogeneity in terms of data. In particular, this divergence is due to: (i) sensors themselves (different hardware with different constraints), (ii) data periodicity (regular - sensors - or based on citizen willingness - mobile app), (iii) data accuracy (depending on sensors' hardware and data frequency), and (iv) data coverage (city' areas may not be uniformly covered). Moreover, to avoid losing of information because of too much noisy data, it is important to consider reliable data-sources of interest for a given application. The aforementioned interest and reliable properties may change over time, therefore the design of efficient adaptable and tuneable content-based data discovery [petrolo,2016,march] and filtering [petrolo,2017] is required. Another important approach for data discovery is the one involving real end users interests based on their profiles, since the adding of human dimension (perception) is of paramount importance for the effectiveness of Internet of Things (IoT) solutions in the context of Smart City [24] since applications aim to provide the citizen more comfort. If for instance people in a room are cold, heating should be switched on even if sensors detect a proper temperature. The human perception must be part of the decision.

\section{Network}

Deployed networks, either wired or wireless, public or private, can be used to collect data depending on the sensors' type and the application requirements. Mobile apps, for example, can rely on cellular or WiFi networks, while distributed wireless sensors may use either traditional cellular networks, or long-range technologies such as LoRA, SigFox, or short-range loT wireless technologies such as Bluetooth, ZigBee, etc. In addition, a single data-source device can be equipped with several communication technologies and conveniently use them according to network availability, rate, range, energy consumption, data-priority, etc. However, due to the huge amount of devices connected and the medium scarcity, new communication protocols are expected in order to properly convey data to data centers, by leveraging for example on multihop paths. Also, due to the variety of applications and networks to be deployed, an interesting point would be for these networks to cooperate and relay opportunistically data from another network. This would relieve the infrastructure and reduce deployment costs and coverage holes. But of course, this comes with new challenges related to the priority of the data for which the network is initially devoted but also in terms of security and flows isolations.

\section{Servers, clouds, and databases}

Data is collected with the aim to better understand the city and its citizens life, and to take actions consequently. For instance, combining the number of visits of a site with its 
environmental data, or the dangerousness of a place with its lighting, could lead to the decision of adding green areas or to better adapt public street lighting. To this end, data needs to be stored over long periods and processed, which will require powerful servers, and databases. In this context, issues are related to data's quantity and to city's policies on the data lifetime. The processing of data is not a trivial task and requires some big data, machine and deep learning approaches to sort, filter, and understand them.

\section{Software applications and data visualisation tools}

Data available on databases can be accessed via API and used to build software applications that talk and shed light on specific aspects of the city and citizens life. Therefore, it is important to share - in an understandable way - this information with citizens and all city stakeholders. To this end, visualization and representation tools are required together with pedagogical approaches so that one can be informed about city dynamics and act consequently.

Thanks to recent advances in software development and thanks to the rise of open sources communities, applications can be directly coded by citizens, which can become active actors in the process of building smart cities. Those applications can be furthermore exposed as a Service - by taking advantage from the everything as a Service paradigm introduced by the Cloud Computing - and used to provide more sophisticated e-services.

\section{Crossed requirements}

All the above described components should be compliant to properties such as energyefficiency, scalability, adaptability, and interconnectivity although the great heterogeneity at several levels (hardware, computing capabilities, communication technology, mobility, energy consumption, density, availability, etc.). Then, these requirements raise new challenges from a technical point of view as well as societal [petrol,2016,june],, data and devices security, together with citizen privacy, should be indeed ensured.

\section{Use cases}

In the following we describe typical scenarios for a smart city. First we describe classical sensing scenarios, in which data sensed can be shared among different applications, then we describe smart networks use cases. These examples are not intended to be exhaustive.

\section{Sensing scenario}

With the fast increase of urban population, cities must face new challenges such as the set up of environmental plans to improve urban noise and air quality. Accredited air and noise monitoring are today measured independently without exploiting their correlation, either with few costly durable measuring systems which deployment is limited by connection availability or by independent distributed initiatives which measures are unreliable. This same information about local air quality levels is also requested by doctors to better understand breath illnesses and by citizens to better plan their walks, especially when they have young children. A will of smart cities is to better measure this air and noise quality for providing better feedbacks to the citizens but also to better plan their urban deployment and associate appropriate traffic plans and green spaces deployment. In this scenario, smart cities can deploy a set of smart sensors 
to monitor different air components and noise levels in specific locations of the city. A wireless distributed network is thus requested to collect all these distributed heterogeneous data.

\section{Transportation Systems}

One of the key challenges for a future city regards its transportation systems. Indeed, due to the increasing number of cars, the traffic flow is often worst. A smart city should look at solutions that can improve traffic management by adapting for example systems, which integrate different data-sources such as street cameras, weather sensors, events, etc. and provide an optimized traffic flow. For example, algorithms can be used to forecast possible accidents and / or congestions by using traffic data together with weather information. Also it has been shown that a fast increase in local air pollution is correlated with a traffic jam. Correlating data from cars and traffic on one hand and from air and noise quality on the other hand can help to better manage the traffic lights for instance.

At the same time, green energy sources should be introduced to reduce air pollution. For example, over the last few years, Lille (France) managed to reduce exhaust gas emission enormously by using natural gas-powered buses [smartcitycouncil] .

\section{Pervasive access}

In urban crowded scenarios many users concentrate in a small area (such as in the case of sport or music events).

In this case, pervasive access needs to be guaranteed by promoting the spontaneous network extension to increase coverage, reliability, and available radio spectrum throughout radio device reconfiguration. In these scenarios, smart networks can help in facing the fragmentation of access technologies and devices by extending the coverage through a mutation in the role of the involved nodes.

Moreover, some network nodes can self-organize and build backbone links to the Internet. The candidates to play the role of egress nodes are the ones provided by the city or by utility/network providers. However, also end-user nodes, e.g., desktop PC, can evolve and become relay nodes or ingress nodes in the backbone network in case of lack of infrastructure. Also other types of end-user devices (e.g., laptops and netbooks) can be part of the backbone network, although at the cost of reduced network performance.

\section{Emergency Applications}

Emergency situations of different natures can occur and damage the network devices/infrastructures and prevent city-wide connectivity.

A smart city solution can effectively manage such a type of situation, by leveraging on "surviving" devices and create a spontaneous communication infrastructure that conveys some basic information to aid diagnosis or to detect dangerous situations [mezghani,2018] .

\section{The Cloud of Things}

In the last few decades, Cloud computing gained attention from both academia and industry across the world thanks to its capability to entirely transform in service model the current Information Technology field. The key advantages introduced by Cloud computing can be summarized as high performance, high availability, infinite scalability, tremendous faulttolerance capability and at the same time, minimization of the upfront investment 
[yuriyama,2010]. Cloud services can be classified according to the offered benefits, for example the Infrastructure as a Service model provides computing resources such as processing and storage, the Platform as a Service allows the development, running, and management of applications without the complexity of building and maintaining the infrastructure [roy, 2017].

Recently, the introduction of the Everything as a Service (XaaS) model [banerise,2011] led the way to a new set of services and scenarios. Indeed, XaaS promotes a "pay as you go" computing, allowing the consumption of a generic service by paying only for the amount of resources used. Due to its economical, efficient, and effective properties, this model results attractive in many IT fields and especially in the context of the loT, where different sensors and actuators are deployed and can be shared and reused without need to deploy new ones [aazam,2014]. In particular, data consumers can access things already available by paying a fee, thus reducing data acquisition costs thanks to the shared nature, and collecting data previously unavailable - due to the business model, companies will be stimulated to "sell" data.

Nevertheless, one main challenge in this context regards the heterogeneity of things [mitton,2012]. Current deployments are indeed based on different standards and protocols, making difficult and challenging the adaption of XaaS for loT. To address this issue, in the last years, a consistent effort has been done on interoperability techniques that allow communication among disparate devices. In [hauswirth2011, march], authors highlight the need of an IP-based access as unifying network layer that can turn those objects into InternetConnected Objects (ICOs). At the same time, authors point out on the need of a semantic representation in order to understand data which comes out and goes into the ICO interfaces. This "data exchange layer" can influence discovery and routing approaches and it will be crucial to enable scalability from an application's point of view, as nobody will be able do deal with the number of ICOs, efficiently and scalable, without such a layer. The technologies to build this layer are already being developed and deployed, Semantic techniques (Linked Data [bizer,2011] , Resource Description Format (RDF) [hayes,2004] ) are indeed well accepted standards in the Web as good enablers in the complex process of integration of heterogeneous data-sources.

Semantic technologies and Cloud computing are at the basis of the Cloud of Things (CoT) paradigm that we introduced in [petrolo-loscri,2017]. The CoT aims to make a better use of distributed resources, to put them together in order to achieve higher throughput and to tackle large scale computation problems. Thus, the CoT enables the horizontal integration of various (vertical) loT platforms, devices, and applications making possible the interoperability between them. 


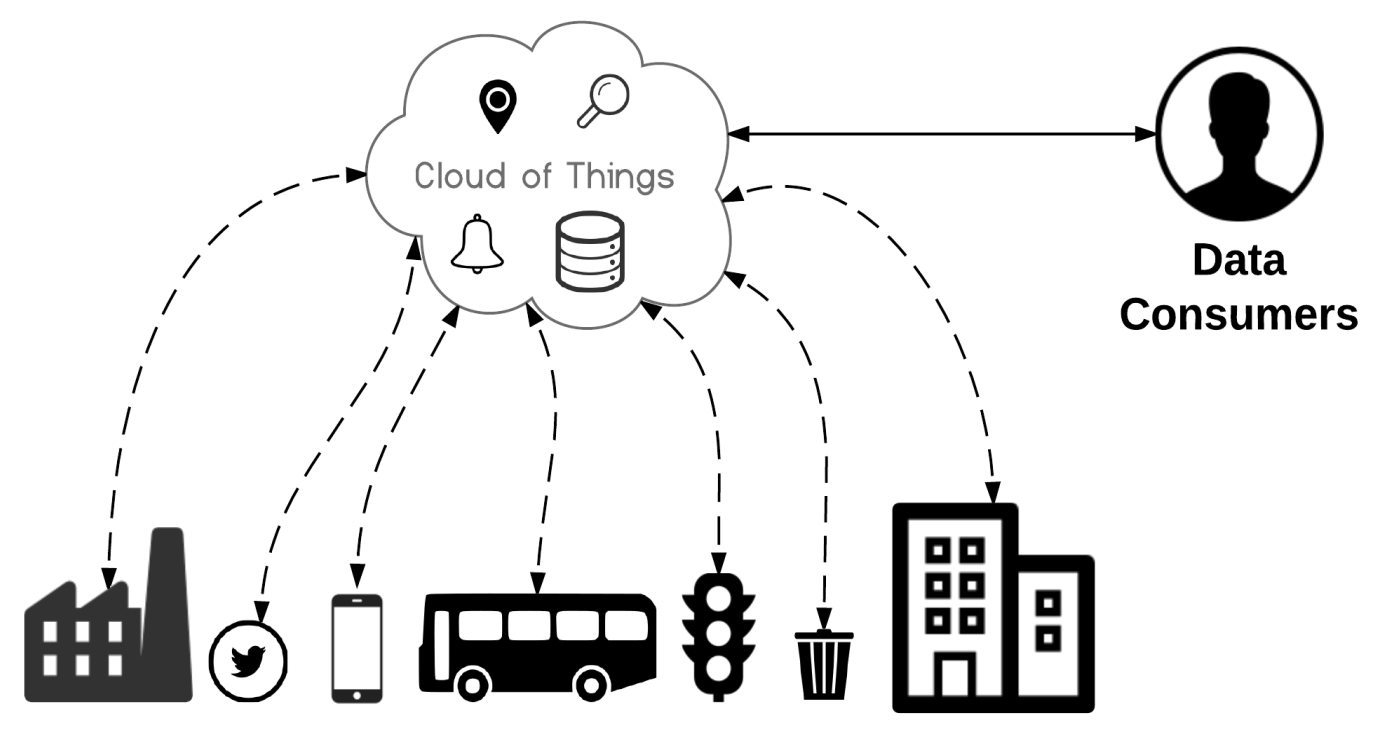

Figure 1: The Cloud of Things

Sensing in cities could range from environmental data sensing (e.g., temperature, air quality, etc.) to human-behavior dependent data (e.g., attendance level of a museum, number of persons in a train or a building, crowdsourcing, etc). This significant spread of scenarios implies a large heterogeneity in devices (sensors, mobile phones, access cards, etc.), mobility (fixed, low/high mobility), communications means (wired/wireless, short range/long range, high/low data rates, etc), and protocols as well in terms of authorities and properties since sensing systems are often deployed independently for each single application, by different administrations. At the same time, as already mentioned, data can be regular (e.g., regular temperature monitoring) or sporadic (e.g., based on citizen willingness through a mobile application), and due to these features (irregularity and mobility) the geographical density of data sources may change over time.

In this context, the CoT can be a great solution and act as a city catalog in which different heterogeneous data-sources are stored and formatted according to semantic reasoning. As shown in Figure 1, data consumers can search and access these resources via a common interface, retrieving relevant information. For example, let us suppose that a user aims to create an application to monitor the speed in a street; without the need to deploy new sensors, he/she can use the CoT to search for devices that provide the requested sensing capabilities (e.g., speed sensor) and are available in the geographical area of interest. At this point, he/she can chose the thing that better suits his/her use case.

As described above, the CoT has strong emphasis on sharing sensed data across different applications and end-users, however it does not deal with smart network aspects, which we will describe in the next section with our vision about the Cloud of meshed cooperative heterogeneous things. 


\section{Vision: from the Cloud of Things to the Cloud of Meshed Cooperative Heterogeneous Things}

The Cloud of Meshed Cooperative heterogeneous Things (CoMCoT) aims to enable every traditional city's entity (node, user, and provider) to be exposed and consumed as a Service. Beyond traditional CoT services such as as data abstraction and mutualization, the CoMCoT provides more holistic functionalities by targeting full interoperability. The CoMCoT is indeed much more than a federation or a set of links between databases and server that store data.

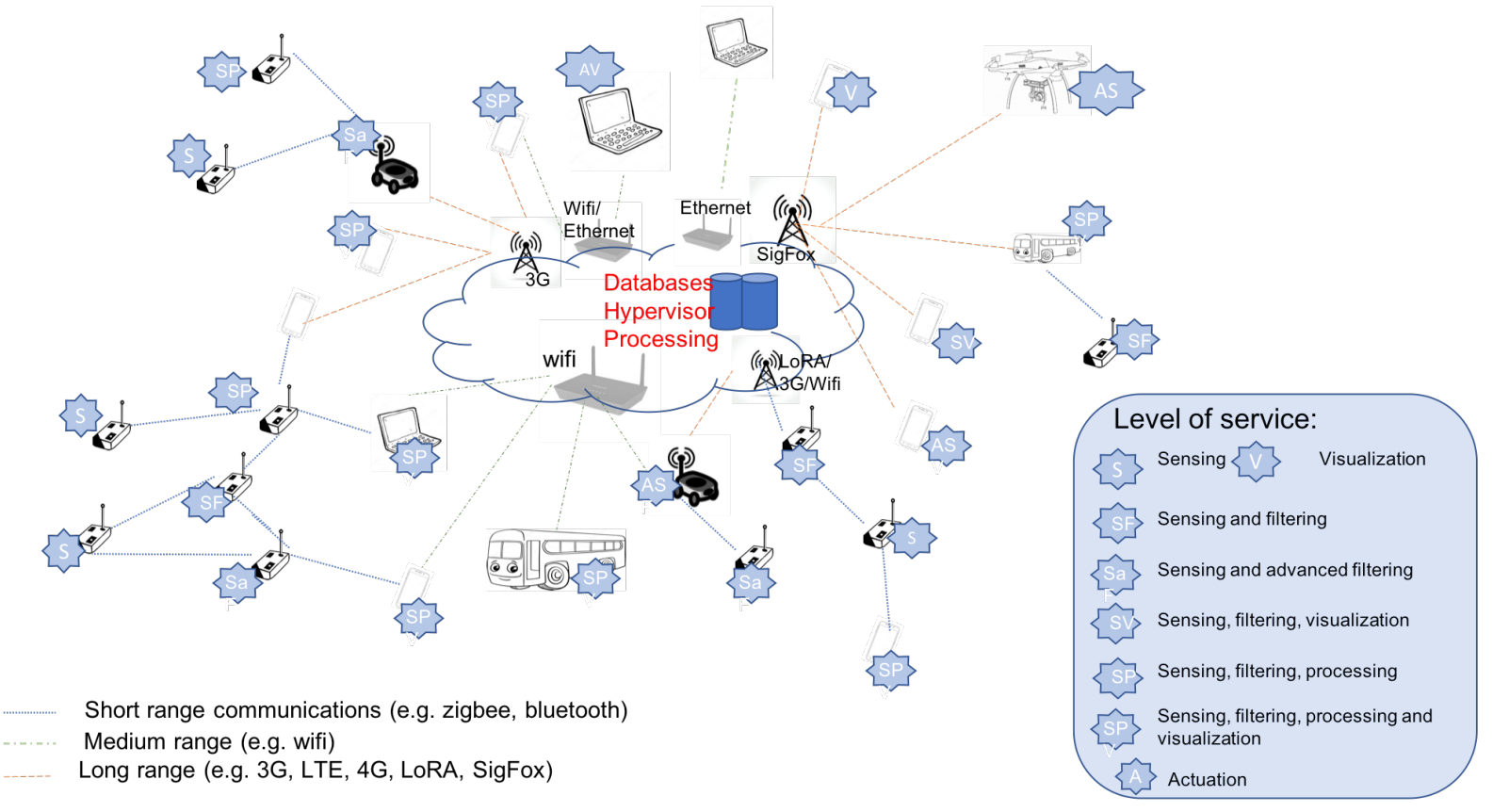

Figure 2: The CoMCOT vision

Figure 2 represents our vision of the CoMCoT. It provides the vision of a flat cooperating multitechnology network composed of heterogeneous devices rather than a juxtaposition of heterogeneous silos. To implement this vision, much research by the scientific community is still needed. The CoMCoT paradigm should provide efficient and adaptive tools for data reduction, prediction, processing, filtering, and visualization tunable for any device that possesses the required features; for instance visualization can only be performed on devices equipped with a screen. These tools should be able to dynamically reconfigure to fit the hardware constraints of each device, environment limitations (e.g., energy, bandwidth, etc.), and application requirements that may change over time. Furthermore, these tools should respect privacy and propose data-priority aware mechanisms.

Single devices such as basic sensors may be used for the only sensing task. More advanced sensors may be able to perform basic filtering mechanisms based on different data reduction rules [razafimandimby, 2017], while a mobile phone will be able to perform more advanced processing and provide a data restitution. These operations could be performed locally, relying only on local data. To get a global picture of the data at the city scale, the device will need to communicate with the cloud that have gathered the entire data set. 
Clouds, servers, and data centres are obviously the most powerful components and are in charge of data consistency, virtualization, and intensive processing. They should provide data to any user/requester with a representation and added-value. Services at this layer can be requested by any device in the city, even by devices that do not necessary hold sensing functionalities but can contribute to the network like a plain router or visualize some data like a screen.

To the best of our knowledge, the CoMCoT vision has not been implemented yet in its globality. It is actually a vision which is difficult to implement over the existing services. Indeed, these latter have been deployed in silos, each network of devices aimed at a specific application and often operated by separated entities. To realize it today, It would be obviously simpler to start from scratch, which is now too late for some places and cities. To integrate existing systems in the CoMCoT vision, there is a need to deploy first a so-king middleware able to mask the heterogeneity of deployed networks and systems and make them communicate and interact in a transparent way, still respecting policy and access rules of each of them. This is for instance what has been developed in the European VITAL project ${ }^{1}$, applied over the Camdem district in London and the city of Istanbul. Then, such a middleware can be extended with the CoMCoT components. Due to its distributed nature, the system can run on the Cloud and on-premises servers of a municipality. We indeed envision that such a system should be managed by a public authority to preserve data privacy.

\section{Enabling functionalities and tools}

During the last years, a lot of research has been conducted on self-configuring distributed systems, technologies for reconfigurable devices/networks, and methods to provide the wireless nodes with cognitive and intelligent capabilities. Most of these solutions can be used for the realization of the core components of a CoMCoT, as discussed in the following text.

\section{Data discovery and selection}

Within the CoMCoT context, one key challenge is to find appropriate services that satisfy user requirements, with more than 50 billion of devices attended to be connected to the Internet in the next few years, it is crucial to have mechanisms capable to provide, in an efficient way, resources that better suit business criteria defined by data consumers. Adaptive and userrelated filters should be set to avoid noisy data that will jeopardize user satisfaction by drowning them in useless information.

The data source discovery is an operation that can be performed directly in-network (i.e., the detection of neighbours) and/or out-network (i.e., a middleware that aims to discover resources that belong to different networks). In the out-network discovery [petrolo,2016,may],

\footnotetext{
${ }^{1}$ vital-iot.eu/
} 
the challenge concerns the representation of different data-sources and how users can easily access those resources and the provided services. Generally, those loT services, are published into registers that are available as end-points of loT platforms.

The in-network discovery process is more related with the building and maintenance of the network itself, especially in order to introduce new services and devices. Data discovery is a known mechanism well used in every wireless ad hoc network. However, very few data discovery mechanisms propose a user-aware data source discovery, considering data pertinency and interest as a function of its geographical position, age, user-preferences, etc, while, as mentioned, it is a key factor to avoid annoying the user with useless data and buy their acceptation of the service. An example of in-network relevant source discovery is CACHACA [petrolo,2016, march].

The advantage of in-network discovery compared to out-network discovery, i.e., to filter data as closely as possible to the source is that less data is transmitted, alleviating the energy and bandwidth consumption of the network. Also, if less data is transmitted, security risks are reduced. The drawback is that data are really customized and are less likely to be reused to answer a request for another user, generating another traffic flow.

A real challenge is thus to determine a tradeoff between the amount of data to be collected in one round and the number of interrogations.

\section{Data collection}

The CoMCoT should provide efficient data collection mechanisms. At this level, challenges are multiple. Indeed, applications use user-generated content from heterogeneous fixed and/or mobile devices gathered or sent in collaboration with its owner/operator with respect to different access rights policies. As already mentioned, such a model requires fundamentally novel algorithms for the data collection, aggregation, filtering, analysis, and composition of different services.

Each device may have different communication interfaces that have to be efficiently combined to optimize the device resources but also release the network occupation. A single and straight forward solution would be that each device uses its medium/long range communication such as $\mathrm{WiFi}, 2 \mathrm{G} / 3 \mathrm{G} / 4 \mathrm{G}$, LoRA to upload its data. But these technologies are generally energy consuming and above all, they come to face the medium scarcity problem. So data communication approaches should feature adaptive cross-layer Medium Access Control (MAC) / PHY layer mechanisms that are able to dynamically and opportunistically select the technology to use but also the PHY layer parameters (frequency, modulations, spreading factors, transmitting power, etc) to increase the medium occupation without degrading the performances of the primary users. This has to be done as a function of data content priority and device remaining resources (in terms of energy and memory).

Even with the large deployment of open communication technologies in cities, they may remain areas that are not covered. Also, some devices may be equipped with an alternative communication technology (such as wireless sensor networks) and are not in range of a base station able to collect data. Then, we require multihop communications with efficient routing protocols. These routing protocols are in charge to determine the path and the intermediate relay devices of a data between its source device to the destination. Challenges arise here 
because of device heterogeneity in terms of communication and computing capacities but also because of the highly dynamic network topology. Indeed, in a CoMCoT case, the network is composed of both fixed and mobile devices that can sporadically and arbitrarily appear and disappear. In every cases, the data collection should be done through seamless communication flows between heterogeneous devices, hiding the complexity of the end-toend heterogeneity from the communication services. The selection of the next relay together with the communication technology to be used to reach it must be carefully done in a local and distributed way with respect to device capabilities and data priority. The different routing protocols should provide a high level of interoperability and a certain degree of flexibility. Today, existing routing protocols do not consider at once all these characteristics and there is still a need for further investigation.

\section{Data processing}

After identifying a data source and raw data is collected, there is need to process it. In a CoMCoT, the goal is not only to process data independently, provider by provider, data kind by datakind, etc. but to give them additional value by crossing different data and exhibit some correlation. The goal is to create new knowledge by learning about citizen habits, climatic changes, etc. and understand the potential correlation between this data. This allows a better urban management and eventually positive changes to make the city safer, more pleasant to live, more sustainable, more respectful of nature, etc. To do so, all data should be crossed and shared between applications, end-users and authorities, with different levels of authorization and access rights. This raises again new challenges in terms of heterogeneity management, interoperability and security issues. Heterogeneity is generally addressed with ontologies (see next session), while interoperability and security have still several facets and can be addressed via standardization (see section $5 \mathrm{~g}$ ).

Where to process data is also a non-trivial question. As for discovery, the closer to the source the data is processed, the less pressure on the architecture ressources and security threats but the less information to be exploited and potentially reused. There is not a single-answer and the best place where to process of data depends of the data, the services and also on the device hardware. As shown in Figure 2, devices feature different hardware characteristics and thus, data processing should be done according to device capacities.

\section{Knowledge representation}

The CoMCoT should provide a homogeneous view of heterogeneous data as well as to provide a sense to it. However, due to the still increasing number of data sensed and transmitted and devices deployed in a more and more limited environment from a technical perspective (data storage, data processing, energy, scarcity of wireless communication medium, etc.), a CoMCoT must go much further beyond this already well adopted vision. A node deployed in the city needs a large set of data to control its operations, including information about the environment, the experience from previous contexts, and the feedbacks from other nodes belonging or not to the same infrastructure.

Data can be made available by different CoMCoT components, therefore methodologies to represent, share, and access it in a uniform and efficient way must be considered for the design of a CoMCoT node. To this purpose, ontologies have emerged as a powerful technique 
to represent semantic knowledge and the relation among concepts in a non-ambiguous way [filipponi2010,july] . Moreover, semantic engines can be implemented on top of ontologies to perform inference from stored knowledge in order to produce new data, which in turn can be used by the core of the CoMCoT infrastructure to guide the reconfiguration process.

\section{Control and decision}

Control functionalities for the core of the CoMCoT are enabled by decision-making algorithms that guide the reconfiguration process of each device. In the case of a multi-radio node, for example, equipped with $\mathrm{Wi}-\mathrm{Fi}$ and $4 \mathrm{G}$ radio interfaces, the core could determine the timeschedule activation of the $\mathrm{Wi}-\mathrm{Fi} / 4 \mathrm{G}$ radios and whether the device should mutate and temporarily serve as a Wi-Fi router (e.g., to extend the access network coverage). Among the approaches proposed in the literature, machine learning techniques [clancy,2007] fit well the need of a smart city device and are useful to determine the optimal configuration/role on the basis of current and previous experiences of the node itself. More specifically, Reinforcement Learning (RL) algorithms [chowdhury,2011,june] are suitable decision making strategies, because of the explicit representation of state/action pairs and the adaptiveness to changes in the environment. Moreover, the RL paradigm can be extended to a cooperative scenario in which devices exchange the information learnt so far and collaborate to the implementation of system-wide policies and network behaviours. Genetic algorithms represent another wellinvestigated area for self-organizing systems that can be used to drive the selection/discovery of the optimal configuration of transmitting parameters [clancy,2007] .

\section{Cooperation and Smart City network creation}

In nature, cooperation is known as an effective strategy to implement system-wide optimization and enable self organization of groups of individuals (e.g. ants and bees) with limited capabilities [[dressler,2010], [miorandi,2010]]. This is also the case of a smart city network composed of heterogeneous devices with limited communication and computation capabilities, which however can accomplish complex tasks through cooperation with other nodes. In a Smart City network, node cooperation techniques are investigated for the purpose of (i) self-optimization, that is, nodes exchange information regarding their own characteristics (such as motion capability, channel quality, and residual energy) and context parameters, in order to reduce the uncertainty about the environment and speed up the role learning process [clancy,2007] and (ii) network creation, that is, nodes adapt their role on the basis of networkwide metrics and requirements such as maximize network coverage and increase reliability.

\section{Location of functions and architecture components}

The complexity of technologies in a CoMCoT, the lack of interoperability and the increasing medium scarcity requires to lower cloud services. It has already been investigated at the edge level (fog computing) such as in [petrolo,2017, zhang,2018, ,liu,2017]. But again, it should also be performed, when the hardware capacities allow for it, at the thing level itself. The main difficulty holds in 1) the determination of the data to send that should be as low as possible for 
a fast processing, a minimum cost in terms of storage, energy and bandwidth but still consistent enough to ensure usefulness and new information as it has already been mentioned and 2) in the service deployment (where and how much data should be processed and/or stored). This latter point is currently a hot topic and relates to SDN (Software Defined Network). Nevertheless, current SDN do not fit the dynamic features of IoT and CoMCoT devices that are potentially mobile, with more or less limited resources and with different confidence and trust levels and can thus not be applied as is.

\section{Standardization}

Standardization activities for Smart Cities play a key role to ensure effective and well-working solutions based on interoperability reduction of the costs and a fair management of the resources. Moreover, standards could pave the way for introducing city indicators in order to characterize the "smartness" of a city.

Another fundamental aspect for considering standard activities is that it could be helpful to clarify guidelines to deploy effective solutions for the cities by considering the market potentiality.

Hence, the premises clearly show the importance of standardization activities, but cities are complex systems and it is not trivial at all to implement standards for such a type of systems. Among the main initiatives regarding the European standards for Smart Cities, we can consider those carried on by the CEN-CLC-ETSI Sector Forum on Smart Cities and Communities. In particular, they have created a list with the most important standardization activities and a living document that can be accessed by any user to be interested to contribute.

In particular, on October 2017, there has been a workshop on "Cities set Standards to be Smarter and more Sustainable" in Brussels, involving city authorities, representative of smart city projects, associations of cities, stakeholders, etc.

The main objective of the workshop was to highlight the importance to define the needs for cities with two different but maybe convergent perspectives: cities' and standardizers'.

The outcome of the workshop has been collected in the CEN-CENELEC-ETSI Forum on "Smart and Sustainable Cities and Communities as advisory and coordinating body for European standardization activities. More information in these activities can be found on: https://www.cencenelec.eu/News/Events/Pages/EV-2017-033.aspx

Yet, standardization seems mandatory to reach global holistic services for a complete smart city but this is not an easy way to make all perspectives converge. However, all stakeholders are aware of this and work for it.

\section{Socio-economic challenges}

Although intimately linked, two main categories of challenges can be envisaged in the context of smart city architectures: technical challenges and socio-economic issues. 
Technical challenges have been already addressed in this chapter and are mainly related to security and privacy issues and interoperability of different architectures and nodes. Security issues are related to data of citizens that can be processed in order to improve the services offered to the final users.

The great effort to be addressed consists into the definition of security approaches based on the vast amount of literature that takes into consideration the unique peculiarities of the Smart City architecture and to design security-by-design solutions.

One of the most "not technical" complicated aspects to be managed in the context of a Smart City concerns the presence of multiple stakeholders with different (even opposite) interests and the involvement of municipalities, citizens, etc. Indeed, the complex scenario derived from the presence of different actors with potentially different interests, could make difficult the practical realization of efficient Smart City solutions.

Most of Smart City architectures are based on the exploitation of data originated from several and different types of devices: sensors deployed in the city, users handheld devices (e.g. smartphones), loT platforms, etc.

Based on these considerations it is necessary to clearly define and address specific aspects such as:

- Who will be in charge for providing the data;

- Who will provide and maintain the architecture and be responsible in case of failure, data lost, data corruptions, etc;

- How customers can be encouraged to provide their devices for Smart City application, namely create incentive to improve the citizen participation;

- Which business models can regulate the relationships among the different Smart City actors (municipality, content and service providers, end-users, companies, etc.)

Economic issues should be seriously considered for Smart City applications. Indeed, Smart City applications certainly require some costly devices in order to face to the software reconfiguration capabilities of the Smart City platforms. Indeed, a software defined approach is a viable way for interaction among already existing loT platforms, or devices that are conceived to be used as handheld personal devices (e.g., smartphones) can be enriched with features to respond to new "Smart City" functionalities. Of course, the benefits need to overcome the costs in order to make the Smart City platform an effective solution.

In order to give an insight of the potential advantages, we can imagine a scenario where buses need to change route due to the detection of an anomalous event.

To face this situation, it would be convenient to use some residential access points, along the new path by dynamically reconfiguring them via software under the control of a "collective intelligence".

A municipality does not need, in this specific case, dedicated network equipment, thanks to an effective Smart City architecture.

Finally, the main lever is the acceptation of the technology/ies and services by citizens. They are a complete part of the system since they will be simultaneously data providers and data consumers and, as the case may be, their personal devices may be used (temporarily and sporadically) as a part of the core architecture. To ensure their cooperation and adoption, 
smart city application and infrastructure must go with communication and feedback from and towards the citizen that should be confident in the service (hence security and safety measures) and see their own interest. This is a paramount element of a Smart City to make it persistent and sustainable. But this is not a trivial task since people acceptation or fears depend of their background, origins and cultures and their criteria differ from one country to another one and according to their age. Therefore, Smart City is a multidisciplinary concept and a global architecture must be designed collectively by gathering computer sciences, hardware manufacturers, sociologists, economists and politics.

\section{Conclusion}

This chapter intends to shift the boundaries towards a new concept wider than the now traditional Cloud of things: a cloud of meshed cooperative heterogeneous things. As in the cloud of things, sensors and actuators not only can be discovered and aggregated, but also dynamically provide as a service, applying the Cloud provisioning model. But, much beyond, the CoMCoT considers all communicating devices as a potential service provider or consumer, but also as an opportunistic network infrastructure node, with respect to its hardware capabilities, data and devices security and privacy requirements, considering them. This has to be done in a seamless way for the user but is required to face the new challenges raised by the integration of ever increasing number of heterogeneous devices.

The CoMCoT's primary use case application is Smart City where more and more devices and services are deployed. To this purpose, all CoMCoT primitives must comply with citizen expectations and needs.

In this chapter, we identify and outline the roadmap to implement this challenging vision. A high-level modular architecture has been defined, identifying blocks to deal with all the issues herein discussed. Such architecture offers data gathered from many heterogeneous devices to Internet clients in a uniform way, by using an abstraction layer.

We outline the strong requirements to design new services that rely on protocols that are secured and privacy-by design and that consider multidisciplinary aspects (networks, electronics, data science, machine learning, data mining, sociologist, politics, economics, etc). It is also very important to involve the citizens from the beginning on the service and application deployment by providing them data feedback and keeping them updated to ensure their acceptation and thus the service success and durability.

In order to be able to accomplish the objectives considered as primary in this chapter, we consider "collective intelligence" as a key factor enabling high levels of efficiency and information accuracy. Collective intelligence is based on the aggregation and prioritization of the perspective of the citizen and their needs and is realized by the means of heterogeneous devices masterfully orchestrated in order to get new data content for offering new services. This collective perspective is the key solution for an urban empowerment, by defining both the problems and solutions through a collective perspective.

Many topics are still open problems and challenges, thus material for future work. 


\section{References}

[petrolo, 17] Petrolo, R., Morabito, R., Loscrì, V., \& Mitton, N. (2017). The design of the gateway for the cloud of things. Annals of Telecommunications, 72(1-2), 31-40.

[ni,17] Ni, J., Zhang, K., Lin, X., \& Shen, X. S. (2017). Securing fog computing for internet of things applications: Challenges and solutions. IEEE Communications Surveys \& Tutorials, 20(1), 601-628.

[liu,17] Liu, Y., Fieldsend, J. E., \& Min, G. (2017). A framework of fog computing: Architecture, challenges, and optimization. IEEE Access, , 25445-25454.

[citypulse,2014,may] FP7 CityPulse European Project, (2014,May). Smart City Use Cases and Requirements. Deliverable D2.1.

[roy, 2017] Roy, S., \& Sarddar, D. (2017). The Role of Cloud of Things in Smart Cities. arXiv preprint arXiv:1704.0705.

.[aazam,2014] Aazam, M., Khan, I., Alsaffar, A. A., \& Huh, E. N. (2014, January). Cloud of Things: Integrating Internet of Things and cloud computing and the issues involved. In Proceedings of 2014 11th International Bhurban Conference on Applied Sciences \& Technology (IBCAST) Islamabad, Pakistan, 14th-18th January, 2014 (pp. 414-419). IEEE.

[petrolo,2017,may] Petrolo, R., Loscri, V., Mitton, N., \& Herrmann, E. (2017, May). Adaptive filtering as a service for smart city applications. In 2017 IEEE 14th International Conference on Networking, Sensing and Control (ICNSC) (pp. 525-530). IEEE.

[petrolo,2016,june] Petrolo, R., Roukounaki, A., Loscri, V., Mitton, N., \& Soldatos, J. (2016, June). Connecting physical things to a SmartCity-OS. In 2016 IEEE International Conference on Sensing, Communication and Networking (SECON Workshops) (pp. 1-6). IEEE.

[petrolo,2016,march] Petrolo, R., Loscri, V., \& Mitton, N. (2016, March). Confident-based adaptable connected objects discovery to HArmonize smart City Applications. In 2016 Wireless Days (WD) (pp. 1-6). IEEE.

[mitton,2012] Mitton, N., Papavassiliou, S., Puliafito, A., \& Trivedi, K. S. (2012). Combining Cloud and sensors in a smart city environment.

[razafimandimby, 2017] Razafimandimby, C., Loscri, V., Vegni, A. M., Aourir, D., \& Neri, A. (2017). A Bayesian approach for an efficient data reduction in loT. In Interoperability, Safety and Security in IoT (pp. 3-10). Springer, Cham.

[yuriyama,2010] Yuriyama, M., \& Kushida, T. (2010). Sensor-Cloud Infrastructure-Physical Sensor Management with Virtualized Sensors on Cloud Computing. NBiS, 10, 1-8.

[banerise, 2011] Banerjee, P., Friedrich, R., Bash, C., Goldsack, P., Huberman, B., Manley, J., ... \& Veitch, A. (2011). Everything as a service: Powering the new information economy. Computer, (3), 3643.

[hauswirth2011, march] Hauswirth, M., Pfisterer, D., \& Decker, S. (2011, March). Making internetconnected objects readily useful. In Interconnecting Smart Objects with the Internet Workshop, Prague.

[bizer,2011] Bizer, C., Heath, T., \& Berners-Lee, T. (2011). Linked data: The story so far. In Semantic services, interoperability and web applications: emerging concepts (pp. 205-227). IGI Global.

[hayes,2004] Hayes, P. (2004). RDF Semantics. Available at: http://www.w3.org/TR/ 2004/REC-rdf$\mathrm{mt}-20040210$.

[petrolo,2016,may] Petrolo, R., Bonifacio, S. G., Loscri, V., \& Mitton, N. (2016, May). The discovery of relevant data-sources in a smart city environment. In 2016 IEEE International Conference on Smart Computing (SMARTCOMP) (pp. 1-5). IEEE. 
[filipponi2010,july] Filipponi, L., Vitaletti, A., Landi, G., Memeo, V., Laura, G., \& Pucci, P. (2010, July). Smart city: An event driven architecture for monitoring public spaces with heterogeneous sensors. In 2010 Fourth International Conference on Sensor Technologies and Applications (pp. 281286). IEEE.

[clancy,2007] Clancy, C., Hecker, J., Stuntebeck, E., \& O'Shea, T. (2007). Applications of machine learning to cognitive radio networks. IEEE Wireless Communications, 14(4), 47-52.

[chowdhury,2011,june] Chowdhury, K., Doost-Mohammady, R., Meleis, W., Di Felice, M., \& Bononi, L. (2011, June). Cooperation and communication in Cognitive radio networks based on TV spectrum experiments. In 2011 IEEE International Symposium on a World of Wireless, Mobile and Multimedia Networks (pp. 1-9). IEEE.

[dressler,2010] Dressler, F., \& Akan, O. B. (2010). A survey on bio-inspired networking. Computer Networks, 54(6), 881-900.

[miorandi,2010] Miorandi, D., Yamamoto, L., \& De Pellegrini, F. (2010). A survey of evolutionary and embryogenic approaches to autonomic networking. Computer Networks, 54(6), 944-959.

[petrolo-loscri,2017] Petrolo, R., Loscri, V., \& Mitton, N. (2017). Towards a smart city based on cloud of things, a survey on the smart city vision and paradigms. Transactions on Emerging Telecommunications Technologies, 28(1), e2931.

[24] Nati, M., Gluhak, A., Abangar, H., \& Headley, W. (2013, June). Smartcampus: A user-centric testbed for internet of things experimentation. In 2013 16th International Symposium on Wireless Personal Multimedia Communications (WPMC) (pp. 1-6). IEEE.

[smartcitycouncil] Smart Cities Council - "How Lille is going green with natural gas-powered buses". Available online at https://eu.smartcitiescouncil.com/article/how-lille-going-green-natural-gas-poweredbuses

[mezghani,2018] Mezghani, F., \& Mitton, N. (2018). Opportunistic disaster recovery. Internet Technology Letters, 1(2), e29.

\section{Biography}

Valeria Loscri is a permanent researcher of the FUN Team at Inria Lille-Nord Europe since 2013. From December 2006 to September 2013, she was research Fellow in the TITAN Lab of the University of Calabria, Italy. She received her MSc and $\mathrm{PhD}$ degrees in Computer Science in 2003 and 2007, respectively, from the University of Calabria. Her research interests focus on heterogeneous communication technologies, their coexistence and complementarity, molecular communication and Visible Light Communication. She has been involved in several european and national projects such as the FP7 European project VITAL, the FP6 European project MASCOT, the national project (PRIN) StemNET and the national project (PON) Kom4t-me. Since 2016, she has been appointed scientific delegate for European partnerships for Inria Lille - Nord Europe.

Nathalie Mitton received the MSc and PhD. degrees in Computer Science from INSA Lyon in 2003 and 2006 respectively. She received her Habilitation à diriger des recherches (HDR) in 2011 from Université Lille 1. She is currently an Inria full researcher since 2006 and from 2012, she is the scientific head of the Inria FUN team which is focused on small computing devices like electronic tags and sensor networks. Her research interests focus on self-organization from PHY to routing for wireless constrained networks. She has published her research in more than 30 international revues and more than 100 international conferences. She is involved in the set up of the FIT IOT LAB platform (http://fitequipex.frl, https://www.iot-lab.info), the FP7 VITAL or H2020 VESSEDIA projects and in several 
program and organization committees such as Infocom 2020\&2019, PerCom2019, Adhocnow 2018\&2016\&2015, ICC 2018, Globecom 2019\&2018\&2017, Pe-Wasun 2017, VTC 2019\&2018\&2017\&2016, etc. She also supervises several PhD students and engineers.

Riccardo Petrolo is currently R\&D Engineer at Konica Minolta Laboratory Europe Rome, Italy.

He received Bs.C. and Ms.C. degrees from University "Mediterranea" of Reggio Calabria, Italy in 2010 and 2013 respectively.

In 2016, he received the Ph.D. degree from Lille 1 University, France. During this period, Dr. Petrolo served as Research Assistant at Inria Lille - Nord Europe, and he was involved in the development of the EU FP7 VITAL project, one of the first Operating Systems for Smart Cities. In 2015, Dr. Petrolo was Visiting Researcher in the GTA group at UFRJ, Rio de Janeiro, Brazil. From January 2017 to January 2019 he was Postdoc Fellow at Rice University (Houston, Texas, USA) where he lead the ASTRO project.

His current research interests include Internet of Things, Robotic Networks, Semantic interoperability, Edge computing, and Smart Cities. 